\title{
Subpicosecond Transient Absorption Studies of the Photocycloreversion of an Aromatic Endoperoxide
}

\author{
N. P. Ernsting, * \\ Max-Planck-Institut für biophysikalische Chemie. Abteilung Laserphysik, Postfach 2481, D-3400 Göttingen, \\ Federal Republic of Germany
}

\author{
R. Schmidt, * and H.-D. Brauer \\ Institut für Physikalische und Theoretische Chemie, Universität Frankfurt, Niederurseler Hang, \\ D-6000 Frankfurt/Main, Federal Republic of Germany (Received: November 13, 1989; \\ In Final Form: February 2, 1990)
}

\begin{abstract}
The photocycloreversion of the endoperoxide of dimethylhomoeocoerdianthrone has been studied by transient absorption spectroscopy. The results are consistent with the current model which assumes a two-step process. In the first step, homolytic rupture of a single $\mathrm{C}-\mathrm{O}$ bond in the reactive $S_{3}\left(\pi \pi^{*}\right)$ state of the endoperoxide takes place within the time resolution of $0.35 \mathrm{ps}$. Thus a transient biradical is formed, which decays thermally with a lifetime of $1.6 \pm 0.5$ ps (at $22{ }^{\circ} \mathrm{C}$ in dichloromethane), to yield ground-state homoeocoerdianthrone and singlet oxygen.
\end{abstract}

\section{Introduction}

Large condensed aromatic hydrocarbons, for example, rubrene, owe their color to an absorption band in the visible range corresponding to the $S_{0}-S_{1}\left(\pi \pi^{*}\right)$ electronic transition. If an aerated solution of the aromatic compound is exposed to sunlight, the color usually fades rapidly. This "photolytic fading" is caused by the insertion of singlet oxygen into the aromatic moiety, thus forming an aromatic endoperoxide. The reactive singlet oxygen is generated by the aromatic hydrocarbon itself through sensitization. In the endoperoxide, delocalization of the $\pi$-electrons can no longer extend over the entire hydrocarbon system. Therefore, the endoperoxide does not absorb visible light.

Conversely, UV irradiation of an aromatic endoperoxide will eject singlet oxygen and regenerate the original aromatic parent compound. This process is termed "photocycloreversion". (See Scheme I.) Kearns and Khan had examined photocycloreversion of endoperoxides in theoretical work assuming that both $\mathrm{C}-\mathrm{O}$ bonds are broken simultaneously in a concerted mechanism. They predicted that the reaction occurs adiabatically from a higher excited $S_{n}$ state. This was corroborated by studies of the quantum yield of the aromatic compound as a function of excitation wavelength ${ }^{2-4}$ and of the product state of oxygen. ${ }^{2-6}$

There are two other main experimental results. First, the lack of any measurable luminescence quantum yield from the photoreactive $S_{n}$ state indicates very fast $(<3 \mathrm{ps})$ deactivation of that state. ${ }^{7}$ Second, the temporal rise of the reegenerated aromatic parent has been followed by two-step laser-induced fluorescence (LIF) in the laboratories of Eisentha $1^{6,8}$ and of Comes. ${ }^{7,9,10}$ It was found that, for most of the investigated endoperoxides, the formation of the aromatic parent compound is a relatively "slow" process with time constants of tens of picoseconds.

These findings may only be reconciled if a two-step mechanism is assumed rather than a concerted one. Thus the rise time of the parent compound reflects the lifetime of an intermediate. As

(1) Kearns, D. R.; Khan, A. U. Photochem. Photobiol. 1969, 10, 193

(2) Schmidt, R.; Drews, W.: Brauer, H.D. J. Am. Chem. Soc. 1980, 102 2791 84.

(3) Drews, W.; Schmidt, R.; Brauer, H.-D. Chem. Phys. Lett. 1980, 70,

(4) Gabriel, R.; Schmidt, R.; Brauer, H.-D. Z. Phys. Chem. 1984, 141, 41

(5) Chou, P.-T.; Frei, H. J. Chem. Phys. 1987, 87, 3843.

(6) Eisenthal, K. B.; Turro, N. J.; Dupuy, C. G.; Hrovat, D. A.; Langan, J.; Jenny, T. A.; Sitzmann, E. V. J. Phys. Chem. 1986, 90, 5168.

(7) Blumenstock. Th.: Comes, F. J.: Schmidt, R. Brauer, H.D. Chem.

Phys. Lett. 1986, 127, 452.

(8) Sitzmann, E. V.; Dupuy, C. G.; Wang, Y.; Eisenthal, K. B. In Picosecond Phenomena: Eisenthal, K. B., Hochstrasser, R. M., Kaiser, W. Lauberau, J., Eds.; Springer-Verlag: New York, 1982; Vol. III, p 168.

(9) Blumenstock, Th.; Jesse, K.; Comes, F. J.; Schmidt, R.; Brauer, H.-D. Chem. Phys. $1989,130,289$.

(10) Jesse, K.; Comes, F. J.; Schmidt, R.; Brauer, H.-D. Chem. Phys. Lett. $1989,160,8$

\section{SCHEME I: Photolytic Fading and Photocycloreversion of an} Aromatic Hydrocarbon (Rubrene)
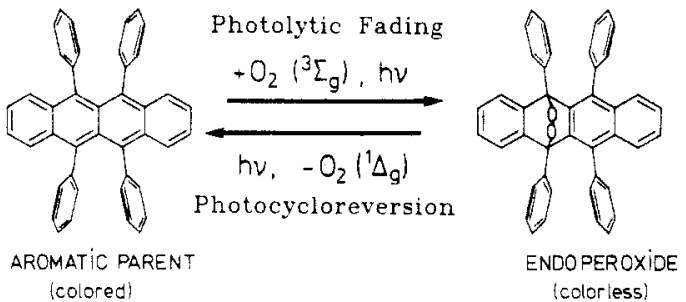

SCHEME II: Two-Step Mechanism of the Photocycloreversion of an Aromatic Endoperoxide

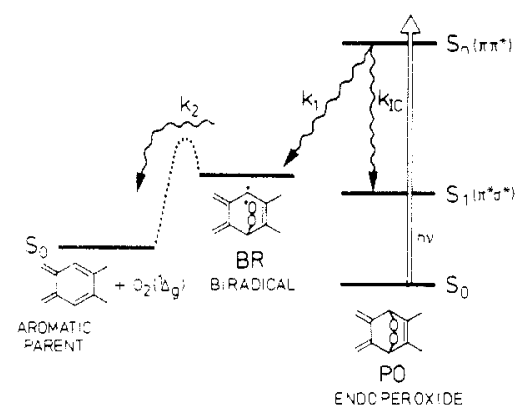

neither polarity nor viscosity of the solvent influences the dynamics of this secondary reaction," the precursor-and product of the primary photochemical reaction-is most probably a biradical (BR) which is formed by homolytic bond rupture. This was first discussed by Eisenthal et al. ${ }^{6}$ and is shown in Scheme II.

The aromatic endoperoxide $\mathrm{PO}$ is excited to a high-lying electronic state $S_{n}\left(\pi \pi^{*}\right)$. Deactivation occurs chemically by fast $\mathrm{C}-\mathrm{O}$ bond rupture with rate constant $k_{1}$, forming the intermediate biradical BR, and physically by internal conversion with rate constant $k_{\mathrm{IC}}$. The $\mathrm{C}-\mathrm{O}$ bond remaining in the biradical is then cleaved in a secondary, thermal reaction with rate constant $k_{2}$.

It was already mentioned that the lifetime $\tau_{2}=1 / k_{2}$ of the hypothetical biradical is normally of the order of tens of picoseconds. However, one exception is the endoperoxide of dimethylhomoeocoerdianthrone (HOCDPO; see inset of Figure 1). From LIF studies only an upper limit of $\tau_{2}<5$ ps could be obtained for the rise time of the parent HOCD. 9 Since in this case $k_{2}$ has so far not been distinguished experimentally from $k_{1}$, it is an open question whether the two-step model does also apply here. We have therefore examined the photocycloreversion of HOCDPO with an overall time resolution of $0.35 \mathrm{ps}$. After optical excitation at $308 \mathrm{~nm}$ with pulse duration of $0.26 \mathrm{ps}$, the induced 
Photocycloreversion of an Aromatic Endoperoxide

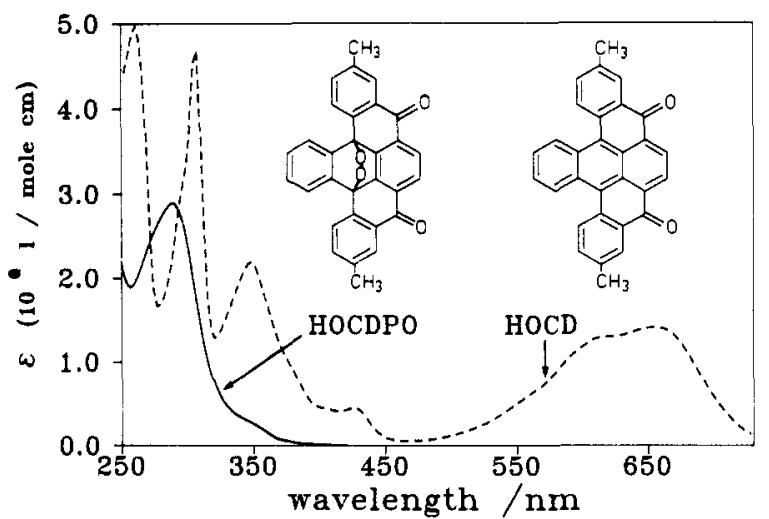

Figure 1. Absorption spectra of dimethylhomoeocoerdianthrone (HOCD) and its endoperoxide, HOCDPO, in dichloromethane.

transient absorption spectrum is recorded in the spectral range 420-730 nm, covering the lowest, characteristic HOCD absorption bands. These measurements complement the earlier LIF studies. They also yield additional information on transients as compared with the more selective two-step LIF techniques, which only detect species fluorescing after excitation.

\section{Experimental Section}

The 100-fs, 616-nm pulses from a colliding-pulse-mode dye laser were amplified in a dye laser amplifier which was pumped by a $\mathrm{XeCl}^{*}$ excimer laser. One part of the amplified beam was frequently doubled; the resulting pulses at $308 \mathrm{~nm}$ were boosted to $1.2 \mathrm{~mJ}$ in a second excimer laser channel. The other part of the diffraction-limited red beam (with pulse energy of $50 \mu \mathrm{J}$ ) was used for white light continuum generation in orthophosphoric acid and silica glass. ${ }^{\prime \prime}$ Pulse durations (assuming Gaussian profiles) for the red and UV pulses were 0.24 and $0.26 \mathrm{ps}$, respectively. The UV pulses could be advanced in time, relative to continuum pulses, by translation of a mirror on a precision linear actuator.

Transient absorption was measured by recording sample and reference spectra simultaneously. ${ }^{11}$ Special care was taken to ensure photometric linearity of the optical multichannel analyzer. The investigated solutions were flown at high speed through a cell with $\mathrm{CaF}_{2}$ windows; the optical path length was $0.115 \mathrm{~cm}$. The sample volume was a fraction of the overlapping volume illuminated by the pump beam. Pump energy density was ca. 2.6 $\mathrm{mJ} / \mathrm{cm}^{2}$, corresponding to $0.4 \mathrm{UV}$ photons $/ \AA^{2}$. The base-line transmission (without pump pulse) was monitored for absorption by accumulating photoproducts. The synthesis and purification of HOCDPO has already been described. ${ }^{12}$ Dichloromethane (Merck UVASOL, additionally purified by column chromatography) at $22^{\circ} \mathrm{C}$ was used as solvent in the photocycloreversion experiments. The concentration of HOCDPO was $3.5 \times 10^{-4}$ $\mathrm{mol} / \mathrm{L}$. Between time scans, the endoperoxide solution was regenerated by ${ }^{\prime} \mathrm{O}_{2}$.

For a determination of time zero for probe wavelengths around $620 \mathrm{~nm}$, ground-state bleaching of malachite green in methanol was used. 11

\section{Results}

Figure 1 shows the absorption spectrum of the endoperoxide HOCDPO and of its aromatic parent (and final product of photocycloreversion) HOCD. Throughout the visible range, only HOCD absorbs. A discussion of the spectrum of HOCDPO in terms of the various singlet states involved is given in ref 13 .

Figure 2 shows transient absorption spectra for the endoperoxide solution after short-pulse photolysis at $308 \mathrm{~nm}$ and with $0.26-\mathrm{ps}$ pulse duration. Spectrum a (open circles) was recorded directly after excitation, at relative delay time $t=0.17 \mathrm{ps}$. Spectrum b

(11) Ernsting, N. P.: Kaschke, M. Submitted for publication. 4909 .

(13) Brauer, H.-D.; Schmidt, R. Photochem. Photobiol. 1985, 41, 119.
The Journal of Physical Chemistry, Vol. 94, No. 13, 1990

5253

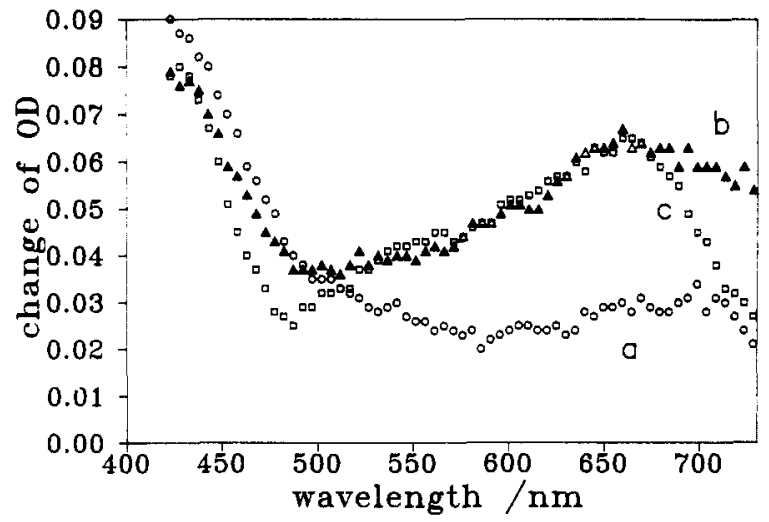

Figure 2. Transient absorption spectra of HOCDPO in dichloromethane: (a) $0.17 \mathrm{ps}$ after photolysis at $308 \mathrm{~nm}$; (b) $10.5 \mathrm{ps}$; (c) $500 \mathrm{ps}$.

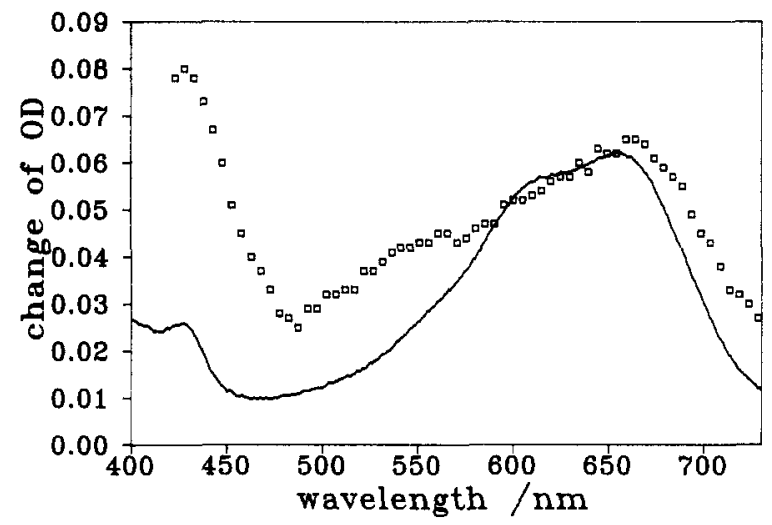

Figure 3. Transient absorption spectrum of HOCDPO in dichloromethane, $500 \mathrm{ps}$ after photolysis. The solid line gives the absorption spectrum of the reaction product HOCD recorded with the same setup and scaled for comparison.

(triangles) was recorded at $t=10.5 \mathrm{ps}$. From that time onward until the largest obtainable delay time of 500 ps (spectrum c, open squares), only gradual spectral changes were noticed. Figure 3 shows again the transient absorption spectrum after $500 \mathrm{ps}$, this time compared with the ground-state absorption spectrum of HOCD, which was recorded with the same setup.

In determining the appearance kinetics of the aromatic HOCD product, we have to consider the possibility that HOCD is generated in its first excited state. In the photocycloreversion of endoperoxides examined so far, ground-state parent hydrocarbons are formed. ${ }^{2,6,7-10}$ However in case that ground-state HOCD is formed ultrafast already during the pump pulse, it will preferentially absorb pump light at $308 \mathrm{~nm}$ owing to its increased absorption cross section at this wavelength as compared to the endoperoxide $\left(\sigma_{\text {purnp }}(\right.$ HOCD $)=1.75 \AA^{2}$, against $\sigma_{\text {pump }}($ HOCDPO) $=0.61 \AA^{2}$; cf. also Figure 1). We have therefore studied separately the excited-state absorption spectrum of the blue HOCD product and find a well-defined isosbestic region between ground- and excited-state absorption centered at $603 \mathrm{~nm}$. At first we choose this wavelength to monitor the appearance of the aromatic hydrocarbon parent HOCD, independent of its being electronically excited or not.

Figure 4 shows the temporal development of transient absorption at $603 \mathrm{~nm}$. The observed change of optical density is generally described by a molecular response function $r(t)$, which is then convoluted with the pump and probe pulses (of fwhm 0.26 and $0.24 \mathrm{ps}$, respectively), and the result is scaled to the observed asymptotic value. It was not possible to fit the experimental data points in Figure 4 by a single exponential rise term, $r(t) \sim[1$ - exp $\{-t / \tau\}]$. Instead it was necessary to assume two rise contributions to the response function, $r(t) \sim\left[a\left(1-\exp \left\{-t / \tau_{1}\right\}\right)+\right.$ $\left.b\left(1-\exp \left(-t / \tau_{2}\right\}\right)\right]$, corresponding to two processes: a first, unresolvably fast one $\left(\tau_{1} \ll\right.$ fwhm $\left._{\text {pump }}\right)$ and a second, resolved process with $\tau_{2}=1.6$ ps. The result (incidentally, $a \approx b$ at $603 \mathrm{~nm}$ ) is 


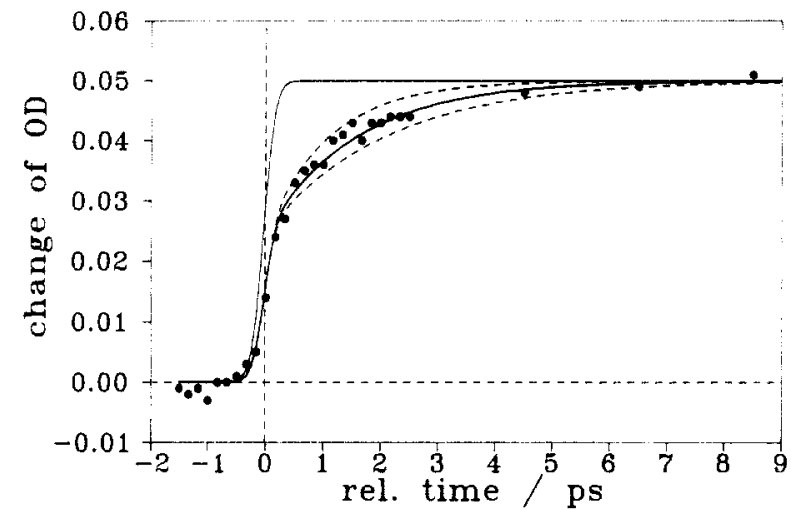

Figure 4. Temporal development of transient absorption at $603 \mathrm{~nm}$, from photolysis of HOCDPO (taken from spectra in Figure 2). Transient absorption due to endoperoxide photolysis is described by two molecular components of equal weight: instantaneous rise and a rise with rise time $\tau_{2}=1.6 \mathrm{ps}$ (solid line through data points). Calculations with $\tau_{2}=1.1$ ps and $\tau_{2}=2.1 \mathrm{ps}$ are also shown (dashed lines). The integrated apparatus function is given for comparison.

shown as a solid, fitted line in Figure 4. This is compared to a curve that would be obtained if the photocycloreversion occurs immediately (i.e., $r(t)=$ constant for $t \geq 0$ and zero otherwise).

In order to estimate the confidence with which $\tau_{2}$ can be determined from our data, two other simulations are shown as dashed lines in Figure 4, with $\tau_{2}=1.1 \mathrm{ps}$ (upper dashed curve) and $\tau_{2}$ $=2.1 \mathrm{ps}$ (lower curve). The data points are well contained between these two limiting simulations. We conclude that $\tau_{2}=1.6 \mathrm{ps}$ is determined with uncertainty less than \pm 0.5 ps.

In Figure 5, the kinetic trace at $603 \mathrm{~nm}$ is compared to traces for $654 \mathrm{~nm}$ and for $450 \mathrm{~nm}$. Notice a shift in time origin; this is due to dispersive effects in the optics of the probe beam. ${ }^{11}$ At $450 \mathrm{~nm}$, the component of instantanteous molecular response is dominant, and the observed rise of $\mathrm{OD}_{450 \mathrm{~nm}}$ follows the calculated convolution of instantaneous molecular rise with pump and probe pulses. (This finding corroborates our assessment of the overall time resolution.) A small decay component has a time constant of $5 \mathrm{ps}$. The rise of optical density at $654 \mathrm{~nm}$-measured at the peak of HOCD absorption-parallels that at $603 \mathrm{~nm}$.

Our experimental results may be summarized as follows. During excitation, broad and unstructured absorption is induced throughout the visible range. In the following, spectral changes occur in two different spectral regions. The most prominent change is recorded in the region $500-730 \mathrm{~nm}$. Here, an absorption band maximizing around $650 \mathrm{~nm}$ rises with a time constant of $1.6 \mathrm{ps}$. In the region $420-500 \mathrm{~nm}$ we observe only a gradual decrease of absorbance to the limiting spectrum recorded at late time ( $500 \mathrm{ps}$ ). The initial part of this decrease is described by a time constant of ca. 5 ps.

\section{Discussion}

Let us return to the two-step model shown in Scheme I. For HOCDPO, the reactive excited electronic state is $S_{3}\left(\pi \pi^{*}\right)$, and an additional singlet state $S_{2}\left(n \pi^{*}\right)$ is located ca. $3000 \mathrm{~cm}^{-1}$ above $\mathrm{S}_{1}\left(\pi^{*} \sigma^{*}\right){ }^{13}$ The lifetime $\tau_{1}=\left(k_{1}+k_{\mathrm{IC}}\right)^{-1}$ determines the appearance kinetics of $S_{2}\left(n \pi^{*}\right)$-excited HOCDPO and of BR. Both processes that deactivate $S_{3}\left(\pi \pi^{*}\right)$ are expected to be very fast. For instance, $k_{\mathrm{IC}} \geq 5 \times 10^{12} \mathrm{~s}^{-1}$ is a reasonable assumption. ${ }^{14}$ In the context of the model, the quantum yield of photocycloreversion is $\Phi=k_{1} /\left(k_{1}+k_{\mathrm{l}}\right)$. For excitation at $308 \mathrm{~nm}$, the experimental value of $\Phi$ is $0.52 .^{13}$ This value indicates that $\mathrm{C}-\mathrm{O}$ bond rupture and internal conversion contribute approximately in equal amounts to the deactivation of $S_{3}\left(\pi \pi^{*}\right)$. Hence we expect a lifetime $\tau_{1}$ on the order of $100 \mathrm{fs}$-well below our time resolution of $0.35 \mathrm{ps}$ (fwhm of pump-probe intensity cross correlation). Therefore, we assign the transient absorption recorded at the early time of $0.17 \mathrm{ps}$ (spectrum a in Figure 2) to the products of the first 381.

(14) Laermer, F.: Elsaesser, T.: Kaiser, W. Chem. Phys. Lett. 1989, 156.

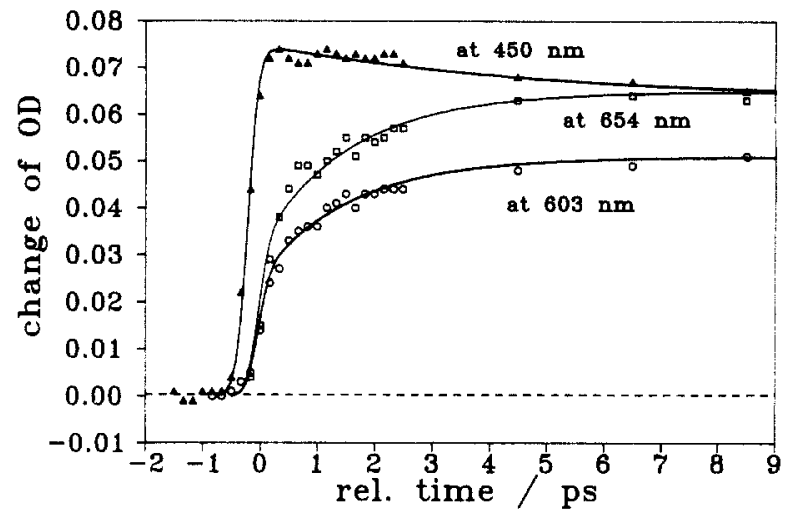

Figure 5. Transient absorption at 450 and $654 \mathrm{~nm}$ due to endoperoxide photolysis. These are compared to transient absorption at $603 \mathrm{~nm}$ from the previous figure. The shift in time origin is due to a difference in group velocity for the three color components of the white light continuum used for the measurement.

reaction step, viz., to a $1: 1$ composition of HOCDPO in some excited state reached via internal conversion and of the biradical BR.

Internal conversion from $S_{3}\left(\pi \pi^{*}\right)$ leads at first to $S_{2}\left(n \pi^{*}\right)$. With regard to the further deactivation of HOCDPO in its $\mathrm{S}_{2-}$ $\left(n \pi^{*}\right)$ excited state, there exists no pertinent measurement at present. In principle there are two possible decay channels: internal conversion to the locally excited $S_{1}\left(\pi^{*} \sigma^{*}\right)$ state of the peroxide chromophore or intersystem crossing to $T_{2}\left(n \pi^{*}\right)$ followed by internal conversion to $T_{1}\left(\pi \pi^{*}\right)$. We can only speculate that the $S_{2}\left(n \pi^{*}\right) \rightarrow S_{1}\left(\pi^{*} \sigma^{*}\right)$ internal conversion occurs probably rather slowly despite the small energy gap of about $3000 \mathrm{~cm}^{-1}$, because of poor spatial overlap of the orbitals involved in this transition. We also expect that intersystem crossing should not be significant on the picosecond time scale. ${ }^{18}$ Thus for the period of 10 ps between spectra $a$ and $b$ of Figure 2, the concentration of $S_{2}\left(n \pi^{*}\right)$-excited HOCDPO may be assumed constant. The prominent spectral change during this time interval is the rise of an absorption band maximizing around $650 \mathrm{~nm}$. This band resembles that of the final product HOCD (cf. Figure 3). Therefore, it is reasonable to assign the prominent spectral change at 650 $\mathrm{nm}$ to the appearance of HOCD. From the appearance kinetics it follows that HOCD has a rise time of $1.6 \mathrm{ps}$. This finding is consistent with the result of a two-step LIF study of HOCDPO, which gave an upper limit of $5 \mathrm{ps}$ for the rise time of ground-state HOCD. ${ }^{9}$ In the context of the model for photocycloreversion shown in Scheme II, the appearance of HOCD is equivalent to the decay of the biradical precursor BR. We conclude that BR has a lifetime $\tau_{2} \approx 1.6 \mathrm{ps}$. If other intermediates occur between $B R$ and ground-state HOCD, then our measurements imply that the lifetime of the biradical precursor BR should be less than 1.6 ps.

Next we assess the contribution of the biradical intermediate to the transient spectra. Generally, the spectrum of a biradical may be approximated by the sum of the spectra of the two constituent radicals. ${ }^{15}$ In the case of HOCDPO, homolytic cleavage of one $\mathrm{C}-\mathrm{O}$ bond should result in an aromatic carbon-centered radical moiety and in an oxygen-centered peroxide radical moiety. The latter absorbs only in the deep UV. ${ }^{16}$ As a model for the aromatic radical moiety, let us take the triphenylmethyl radical: its first adsorption band at $514 \mathrm{~nm}$ has small extinction coefficients $\left(\epsilon_{\max } \approx 656 \mathrm{~L} \mathrm{~mol}^{-1} \mathrm{~cm}^{-1}\right.$, compared to $\epsilon_{654 \mathrm{~nm}}=1.41 \times 10^{4} \mathrm{~L} \mathrm{~mol}^{-1}$ $\mathrm{cm}^{-1}$ for HOCD); at shorter visible wavelengths this is followed by even less adsorption. ${ }^{17}$ We infer that the intermediate BR should not absorb near $654 \mathrm{~nm}$, so that the change of optical

(15) Johnston, L. F.; Scaiano, J. C. Chem. Rev. 1989, 89, 521.

(16) Simic, M.; Hayon, E. J. Phys. Chem. 1971, 75, 1677.

(17) Taub, I. A.; Harter, D. A.; Sauer, M. C., Jr.; Dorfman, L. M. J. Chem. Phys. 1964, 41, 979.

(18) Experiments are planned to clarify the decay of $S_{2}\left(n \pi^{*}\right)$-excited HOCDPO, by following the transient absorption after direct femtosecond excitation at $400 \mathrm{~nm}$. 
density at that wavelength, $\mathrm{OD}_{65 \mathrm{~nm}}=0.035$, between 0.17 and 10.5 ps after excitation must be due only to the formation of HOCD. For the concomitant disappearance of the precursor BR, the equivalent maximum contribution to spectral change can then be estimated at $|\Delta O D| \leq 0.0017$, which is below the noise level of our transient spectra. To summarize so far:

First, the transient spectrum a of Figure 2 originates mainly from a product state of internal relaxation within the aromatic endoperoxide. This product state is populated ultrafast, i.e., even during the pump pulse of $0.26 \mathrm{ps}$; then its population does not change appreciately on a 100 -ps time scale. We speculate that this product state is $S_{2}\left(n \pi^{*}\right)$-excited HOCDPO.

Second, the evolution toward spectrum $b$ and associated with a time constant of $1.6 \mathrm{ps}$ is due only to the formation of HOCD.

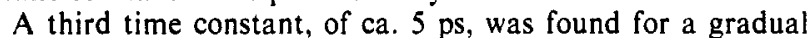
decrease in absorbance at $450 \mathrm{~nm}$. Similarly, a decrease of absorbance at the red side of the main HOCD absorption band was observed (cf. Figure $2 \mathrm{~b}, \mathrm{c}$ ). Both may be explained as a result of cooling of the HOCDPO product state and of hot ground-state HOCD, respectively.

Finally, consider the large variation of rise times $\tau_{2}$, in the range $1.6-75 \mathrm{ps}$, for the photocycloreversion of endoperoxides. ${ }^{6-10}$ It was recently shown by Jesse et al. ${ }^{10}$ for the endoperoxide of heterocoerdianthrone (HECDPO) that the development of HECD is a thermally activated process, with activation energy $E_{\mathrm{a}}=5.7$ $\mathrm{kcal} / \mathrm{mol}$. The very large preexponential factor, $A=10^{14.8} \mathrm{~s}^{-1}$, points to the allowedness of the thermal reaction, which at $22^{\circ} \mathrm{C}$ has $\tau_{2}=40( \pm 10) \mathrm{ps}$. With regard to the present case of HOCDPO, assuming the same preexponential factor but an activation energy that is lower by $1.9 \mathrm{kcal} / \mathrm{mol}$ would account for the 25 -fold shorter rise time $\tau_{2}=1.6 \mathrm{ps}$. Thus the apparent large variation of experimentally found $\tau_{2}$ values could be reduced to a relatively small change in energy barrier for an activationcontrolled process.

\section{Conclusion}

Using HOCDPO as model compound, the photocycloreversion of an aromatic endoperoxide to its aromatic parent has been followed for the first time by transient absorption spectroscopy in the subpicosecond to 10-ps time range. Only two contributing transient spectra are distinguished: that of a primary product state for internal relaxation of the aromatic endoperoxide - most likely $\mathrm{S}_{2}\left(\mathrm{n} \pi^{*}\right)$-excited HOCDPO-and that of the hot product HOCD. The observations are consistent with the current two-step model for the reaction. HOCD is produced in the ground state in a thermal, secondary reaction. Its biradical precursor does not contribute significantly to transient optical absorption in the visible spectral range. The lifetime of the biradical is inferred from the rise time of HOCD absorption to be $1.6 \mathrm{ps}$.

Acknowledgment. We are grateful to Prof. F. P. Schäfer for a critical reading of the manuscript and to the Deutsche Forschungsgemeinschaft for support through the Leibniz Prize Program.

\title{
A KInetic Investigation of the $\mathrm{Ca} / \mathrm{CaO}$ System: Non-Arrhenius Behavior of the Reaction $\mathrm{Ca}\left({ }^{1} \mathrm{~S}\right)+\mathrm{N}_{2} \mathrm{O}$ over the Temperature Range 250-898 K and a Study of the Reaction $\mathrm{CaO}+\mathrm{O}$
}

\author{
John M. C. Plane* and Chia-Fu Nien
}

Rosenstiel School of Marine and Atmospheric Science and the Department of Chemistry, University of Miami, Miami, Florida 33149 (Received: November 17, 1989; In Final Form: February 5, 1990)

\begin{abstract}
A study is presented of the reaction $\mathrm{Ca}\left({ }^{1} \mathrm{~S}\right)+\mathrm{N}_{2} \mathrm{O}$ over the temperature range 250-898 $\mathrm{K}$. Ca atoms were produced in an excess of $\mathrm{N}_{2} \mathrm{O}$ and $\mathrm{He}$ bath gas by the pulsed two-photon $(193.3 \mathrm{~nm})$ dissociation of $\mathrm{CaI}_{2}$ and then monitored by time-resolved laser-induced fluorescence spectroscopy at $\lambda=422.7 \mathrm{~nm}\left(\mathrm{Ca}\left(4^{1} \mathrm{P}_{1}-4^{\prime} \mathrm{S}\right)\right)$. Above $500 \mathrm{~K}$ there is a clear upward curvature in the Arrhenius plot, and the best description of the temperature dependence of the rate constant over the experimental temperature range is given by $k(T)=(2.43 \pm 0.29) \times 10^{-11} \exp \left[-(6.84 \pm 0.27) \mathrm{kJ} \mathrm{mol}^{-1} / R T\right]+(9.70 \pm 1.39) \times 10^{-10}$ $\exp \left[-(24.58 \pm 1.09) \mathrm{kJ} \mathrm{mol}^{-1} / R T\right] \mathrm{cm}^{3}$ molecule $\mathrm{s}^{-1} \mathrm{~s}^{-1}$. Such behavior is explained by vibrationally excited $\mathrm{N}_{2} \mathrm{O}$ enhancing the reaction at high temperatures. This rate coefficient expression, and the chemiluminescence cross section obtained in a previous study of the title reaction in a beam-scattering experiment, are used to estimate a photon yield of $12.5 \%$. This yield is shown to be about half that predicted by a statistical consideration of the densities of states in the reactant electronic channels. In a separate experiment, the two-photon $(193.3 \mathrm{~nm})$ dissociation of $\mathrm{CaO}$, produced by mixing a flow of $\mathrm{Ca}$ atoms and $\mathrm{N}_{2} \mathrm{O}$, is used to study the reaction $\mathrm{CaO}+\mathrm{O} \rightarrow \mathrm{Ca}+\mathrm{O}_{2}$, which proceeds at $(6.5 \pm 2.2) \times 10^{-10} \mathrm{~cm}^{3} \mathrm{molecule}^{-1} \mathrm{~s}^{-1}$ at $805 \mathrm{~K}$.
\end{abstract}

\section{Introduction}

The reaction

$$
\mathrm{Ca}\left({ }^{1} \mathrm{~S}\right)+\mathrm{N}_{2} \mathrm{O} \rightarrow \mathrm{CaO}+\mathrm{N}_{2}
$$

is one of a class of reactions of group 1 and 2 metals with $\mathrm{N}_{2} \mathrm{O}$ that is of both theoretical and practical interest. These reactions tend to be highly exothermic, so that the metal oxide product is often formed in excited states giving rise to strong chemiluminescence. An important theoretical challenge has been to understand the branching ratios for the nascent production of the accessible product electronic states in these reactions, which involve multiple potential energy surfaces. ${ }^{1,2}$ In the case of reaction 1 ,

(1) Alexander, M. H.; Dagdigian, P. J. Chem. Phys. 1978, 33, 13. this chemiluminescent property has been studied extensively, both under single-collision conditions in molecular beams ${ }^{3-7}$ and in diffusion flames. ${ }^{8-13}$ A major practical interest in these types

(2) Yarkony, D. R. J. Chem. Phys. 1983, 78, 6763.

(3) Irvin, J. A.; Dagdigian, P. J. J. Chem. Phys. 1981, 74, 6178.

(4) Dagdigian, P. J. Chem. Phys. Lett. 1978, 55, 239.

(5) Wang, J.; Menzinger, M. Can. J. Chem. 1983, 61, 2703.

(6) Jonah, C. D.; Zare, R. N.; Ottinger, Ch. J. Chem. Phys. 1972, 56, 263

(7) Ottinger, Ch.; Zare, R. N. Chem. Phys. Lett. 1970, 5, 243.

(8) Bernard, D. J.; Slafer, W. D.; Lee, P. H. Chem. Phys. Lett. 1976, 43, 69.

(9) Capelle, G. A.; Jones, C. R.; Zorskie, J.; Broida, H. P. J. Chem. Phys. 1974, 61, 4777 .

(10) Bernard, D. J.; Slafer, W. D.; Hecht, J. J. Chem. Phys. 1977, 66, 1012 .

(C) 1990 American Chemical Society 